\section{Valores y responsabilidades del Diseño en la era de lo "hiper"}

Martha Gutiérrez Miranda ${ }^{(*)}$

Resumen: En un contexto de las prácticas profesionales en mutación, no existe profesión que permanezca estática al cambio tecnológico. En el caso del Diseño, se ha trastocado su concepción, fundamento y acción. Este proceso y la necesidad de transformación de los valores de los diseñadores, obligan a modificar también la manera de asumir la profesionalización de la disciplina y poner atención en el problema epistemológico de la identidad profesional, como representación y auto-concepto.

En un mundo donde todo es "hiper", como dice Lipovetsky (2006), los individuos hipermodernos, aspirantes a diseñadores, quizás estén mejor informados y tengan acceso al conocimiento de forma inmediata, estén predispuestos a la tecnología y "precargados" con gran número de habilidades, aunque en realidad presentan grandes carencias. Paradójicamente, se ha empobrecido la conciencia social y cultural de la profesión, para dar paso a un pragmatismo disociado de la responsabilidad y asociado a la novedad o moda.

Esta reflexión, busca indagar sobre los valores, identidad y ética profesional del Diseño. Analiza la orientación o reorientación de los procesos formativos y la importancia de la ética, para reconcebir al diseñador como sujeto perteneciente al sistema social que, al intervenir en él profesionalmente, lo modifica o renueva.

Palabras Clave: diseño - ética - identidad - profesional - valores

[Resúmenes en inglés y portugués en las páginas 199 - 200]

${ }^{(*)}$ Diseñadora de la Comunicación Gráfica, Maestra en Mercadotecnia y Doctora en Diseño Línea Nuevas Tecnologías. Profesora de tiempo completo en la Universidad Autónoma de Querétaro, coordina la Maestría en Diseño y Comunicación Hipermedial y el Cuerpo Académico Perspectivas Transversales de las Artes. Miembro del Sistema Nacional de Investigadores del CONACYT (México).martha.gutierrez@uaq.mx

\title{
Introducción
}

Una profesión se concibe como una facultad o capacidad adquirida por el sujeto, para realizar un trabajo especializado. Estos conocimientos, el desarrollo de habilidades y la internacionalización de ciertos principios y valores, servirán al grupo social donde se encuen- 
tra inserto. Las profesiones en el mundo representan elementos medulares del progreso y son factores estratégicos del crecimiento de la sociedad, pues empujan y promueven el desarrollo social, cultural, científico y tecnológico, a la par de los cambios en el estilo de vida y las manera en que ésta se va desenvolviendo. A través de su ejercico y quehacer cotidiano van sentando las bases para nuevas y mejores forma de vivir. Hoy, en el sistema de acción profesional, conformado por individuos de perfiles diversos, existe una gran oportunidad de actuar y contribuir a la resolución de problemas y desafíos a los que se enfrentan las sociedades presentes y las venideras.

Según Reyes y Pedroza (2015), la acción profesional es la unidad fundamental del sistema profesional - perteneciente al sistema primario social - y se relaciona directamente con el resto de los sistemas primarios: culturales, conductuales y de la personalidad. La acción profesional es un fenómeno social, ya que considera la interacción entre los actores que en ella participan. Desde esta perspectiva, las profesiones representan un fenómeno social y cultural.

Este mundo de las profesiones sin duda cambia y ha cambiado rápidamente, particularmente en las últimas décadas. Prácticamente no existe profesión alguna que permanezca estática ante el desarrollo tecnológico y, el Diseño no ha sido la excepción, pues se ha trastocado su concepción, fundamento y acción. Todo ello ha sido provocado por esta revolución digital, que demanda un profesional listo para los cambios y no solo habilitado en el manejo de las tecnologías, sino con ideas creativas e innovadoras, que respondan a muchas y diversas necesidades, que encare de forma consciente problemáticas trascendentales y que asuma posturas comprometidas en lo social, cultural y ético, valores de su profesión y exigencias de este siglo.

Ante esta situación Victor Margolin (2011) afirma:

históricamente, la tarea del diseñador era más fácil de lo que es en la actualidad y la responsabilidad del diseñador era más sencilla de definir[...] Actualmente, enfrentados a la creciente complejidad del mundo productivo, los diseñadores deben pensar más profundamente sobre el futuro y sobre cuál es el rol que tienen en traerlo al presente. (p. 76)

Para Adela Cortina (2000), una profesión va más allá de una ocupación que permite obtener ingresos y estatus, es una práctica social que adquiere su verdadero sentido y significado en el bien o servicio que proporciona a la sociedad. En esta misma línea, Augusto Hortal (2002), fundamenta la dimensión ética de la profesión, al señalar que el profesionista, al adquirir los conocimientos y habilidades que lo distinguen como tal, también adquiere el compromiso y la responsabilidad de prestar bien y de manera eficiente sus servicios y conocimientos pues es, a través de ello que la sociedad lo acepta y reconoce como tal.

La profesora Cortina (2009) parte de la idea de que un buen profesional conoce su actividad e identifica cuáles son los bienes internos y las virtudes de la misma, es decir, las excelencias de su profesión. Pero sobre todo, debe estar dispuesto a arriesgarse en su cometido, porque está consciente de la importancia de la labor que realiza y tiene la vocación para hacerla. En este sentido, el profesional se siente llamado a ello, pues asume una identidad más completa al reconocer el valor de su profesión y encarar los retos que representa. La 
gente se siente realizada cuando hace lo que le gusta y puede encontrarle significado a su tarea colaborando en su entorno, ahí es donde verdaderamente radica su importancia y tiene repercusiones positivas en su propia identidad y autoconcepto.

La identidad profesional se logra cuando se llega a ser creativo en el trabajo y no un ejecutante alienado en el rol, permitiendo así desarrollar las actividades en forma óptima, sin dejar de ser persona. Desde esta perspectiva, las profesiones representan un fenómeno de tipo social y de tipo cultural, y la acción profesional de cada especialización y su conocimiento, es más determinante en el actuar de los individuos.

Dado que las profesiones ocupan un lugar relevante tanto en el nivel social como personal, se considera a la ética profesional como condición de posibilidad y realización del bien social y de justicia. Asimismo, en el nivel personal, se vincula fuertemente con los proyectos de vida, la definición del autoconcepto y la autorrealización. En este sentido, hablar de ética no solo es añadir condiciones al ejercicio profesional, más bien centrarla de modo explícito en un campo concreto de la actividad humana, al que aplica su saber fundamental. Si bien existe una marcada tendencia a la elaboración de códigos éticos, se reconoce que la ética de la profesión se aprende al mismo tiempo que ésta y, generalmente, de forma implícita.

Así, un buen profesional conoce sus deberes, alcances y compromisos, aunque no sepa de memoria normas o reglamentos. Bajo esta premisa, la escuela, juega un papel preponderante en esa determinación de la identidad profesional y sus dimensiones éticas. Los valores y la ética profesional - y muy ligado a ellos la identidad-, son preocupaciones que se encuentran en el centro de los debates públicos de la sociedad actual y que se encaminan en la misma ruta de la formación profesional.

\section{Enfoques desde el diseño}

Como disciplina profesional, el diseño se asocia a las disciplinas creativas capaces de proyectar y ofrecer soluciones a necesidades de los usuarios, igualmente, se identifica como un proceso de preconfiguración de soluciones destinadas a producir algo que logre satisfacer necesidades. Es una disciplina proyectual que se orienta a la solución de problemas de comunicación, a través de objetos que faciliten la adaptación de las personas a su entorno.

Santiago Miranda (2009) afirma que "diseñar, como cualquier otra actividad humana, es una actividad compartida. Por ello, requiere el consenso, la colaboración y el esfuerzo de los demás" (p.9). Es una actividad dirigida a satisfacer necesidades, deseos y aspiraciones. Sin embargo, lo paradójico es que se diseña y produce a partir de necesidades que la mayoría de las veces son ajenas al diseñador, sin que esta afirmación se radicalice, pues no hace falta ser músico para diseñar un cartel de un concierto o escritor para diseñar un libro, sino ser un profesional conscientemente responsable de su trabajo y de todas las implicaciones o repercusiones que pudiera tener.

La disciplina se configura profesionalmente durante el siglo XX y, a pesar del reconocimiento relativamente nuevo, en realidad se habla de poco más de 100 años (de la Bauhaus 
a nuestros días) tiene palpable intervención en la vida cotidiana. El diseño se ocupa de proponer, desarrollar, configurar y hasta reconfigurar objetos o productos para dar respuesta a demandas, necesidades y deseos de usuarios reales que las solicitan. En este sentido, la profesión del diseño, el oficio - como firma Norberto Chaves (2006) - se concibe en el plano de la responsabilidad social.

A propósito de ello, el mencionado autor sostiene:

Ha transcurrido ya casi un siglo desde que se acuñara el término "diseño" para denominar a esa entonces nueva práctica productiva. A lo largo de ese período, el diseño ha ido sufriendo una serie de transformaciones que han incidido en la modificación de su propio concepto. Debido a ese dinamismo de cambio, también se ha ido generando una serie de errores de definición, agravados por los usos abusivos del término "diseño". (Chaves, 2006, p.15)

En el texto que acompaña su charla también refiere que, al cabo de varias décadas -relativamente pocas- el diseño se ha transformado en algo muy distinto a aquél de los principios. Hoy día, el diseño invade el consumo masivo y todos los sectores de la industria productiva, la distribución, los servicios, e inclusive, hay un abuso del término, que se ha utilizado como bandera de ese consumismo y que se agrega como calificativo para los productos, lo que lógicamente confunde más los alcances y concepciones del diseño; tanto como de lo que es y hace un diseñador.

El diseñador se constituye como un profesional que interviene en la concepción del mundo para formalizar y dar un servicio útil a empresas y personas, haciendo hincapié en su capacidad para involucrarse en distintos procesos, desde los más técnicos, hasta los más complejos: económicos, sociales, culturales, estéticos, psicológicos, etc., definiendo y desarrollando productos y servicios de diseño para resolver esas necesidades.

Reyes y Pedroza plantean que:

La profesión del diseño es hoy en día un fenómeno sociocultural y tecnológico de gran impacto en la vida cotidiana de los individuos y el desarrollo de todas las naciones. [...] la profesión del diseño se ha convertido en la generación e implementación de estrategias de crecimiento mundial. Impacta no sólo en el resto de la cultura material, el estado del arte de los objetos y el uso de los mismos y de su manera de producirlos, sino de igual forma en la economía, en los modos de distribución, venta y consumo. Impacta al propio ser humano. (2017, p.2)

En este sentido, el diseño se inserta en la vida cotidiana y, sus dimensiones y alcances, trastocan todos los órdenes sociales. Víctor Papanek (1971) declaró que los diseñadores y profesionales creativos tienen responsabilidad social pues su actividad implica cambios en el mundo real, según hagan buen o mal diseño. El mencionado autor, escribe sobre diseño responsable y plantea que los diseñadores tienen responsabilidad sobre las opciones o propuestas resultantes de sus procesos del diseño. De esta forma, se habla de un sujeto perteneciente al sistema social que al intervenir en él desde su actuar profesional, lo modifica o renueva. 
También Víctor Margolin (2017) contribuye con la definición del diseño social, definiéndolo como aquella actividad productiva que intenta desarrollar el capital humano y social, al mismo tiempo que productos y procesos provechosos. Así el diseñador debe prever y dar forma a productos materiales e inmateriales que pueden resolver problemas humanos en amplia escala y contribuir al bienestar social. En esta visión, el diseño social es una actividad profesional y económica, que debe ser vista como una contribución profesional que tiene como fin el desarrollo económico local. De esta manera, trastoca todo el sistema social y sus subsistemas.

Adicionalmente Margolin, muestra a través de diversos escritos, el proceso de cambio que han vivido en los últimos años las profesiones del diseño, y la necesidad de transformación de los valores que hoy deben sustentar a la formación de los futuros diseñadores en el entorno internacional. Afirma atinadamente que:

Actualmente los diseñadores enfrentan situaciones que generan problemas éticos y valores sin precedentes. Las nuevas tecnologías están transformando nuestra relación con el mundo material y con los demás. Ingenieros, programadores, diseñadores de productos y científicos son quienes están en el centro de estas transformaciones. (Margolin, 2011, p.75)

Es decir que la responsabilidad social y los valores éticos acotan y determina aún más el perfil profesional del que diseña. Se entiende al concepto de la responsabilidad social como una especie de obligación, que los miembros de una comunidad tienen para preservar las buenas condiciones de su alreadedor. En efecto, las decisiones que cada individuo realiza, o aquellas que se toman en pequeños y grandes grupos, tienen repercusiones y consecuencias sobre el resto lo que desarrolla una conciencia individual y colectiva, tal como la define Pierre Lèvy (2004). Por ello está tan íntimamente relacionada con la ética y la moral. En este sentido, Norberto Chaves es muy contundente cuanto afirma que "el diseño asume cada vez una mayor responsabilidad en la definición del entorno habitable" (Cháves, 2006, p.14).

La acción profesional del diseñador constituye una clara muestra de distinción e igualdad, de pluralidad e individualidad. En este marco de la ética, el diseño a través de la figura del diseñador es vigía responsable del medio ambiente e integralmente partícipe en todas las esferas y dimensiones del mundo (Reyes y Pedroza).

\section{Desde las escuelas}

Como se mencionó previamente, el Diseño trastoca todo el sistema social y sus subsistemas y es en el subsistema educativo -a través de la enseñanza, el aprendizaje y la investigación- donde encuentra verdadero significado y sentido. En efecto, con la transmisión del conocimiento de las diferentes disciplinas que lo configuran, los profesores contribuyen a formar diseñadores capacitados y habilitados para dar respuesta a las necesidades que en 
su materia les son requeridas por la sociedad, y que cada vez exige más (Reyes y Pedroza, 2017).

A partir de esta concepción, la formación universitaria es un eslabón importante en la cadena de producción. En gran medida depende de ella, pues es la que permite que existan diseñadores, formados con todos los requisitos profesionales, de manera que pueden responder a las necesidades, demandas y exigencias del mercado. Sin embargo, el diseño enfrenta una serie de paradojas, entre las que destaca la irrupción, a veces desmedida, de las tecnologías, no solo en los procesos de producción, sino también en los formativos, o sea, en las escuelas que preparan a los profesionales del diseño. Pues se ha malentendido la incorporación de la tecnología como estrategia para mejorar el propio proceso del diseño, incluso se ha convertido en medio y fin.

La docencia universitaria no solo es complicada por lo que se refiere a formar profesionales, sino por la complejidad que envuelven los saberes que deben comunicarse $y$, en áreas como el diseño, ésta aumenta por las temáticas tan particulares que deben transmitirse en los salones de clase. En casi todas las universidades y escuelas que imparten las licenciaturas en Diseño, (con sus distintos apellidos: gráfico, industrial, textil, de modas, etc.) existe la necesidad urgente de replantear varios de sus enfoques, comenzando por los mecanismos de selección para el ingreso y permanencia, hasta los mecanismos que tienen -o no tienen- para formar profesionales integrales y fomentar con éxito la inserción del egresado en el campo laboral y las áreas productivas.

Con respecto a la educación o propiamente a la enseñanza del Diseño, uno de los problemas más serios es que no se enseña la verdadera importancia social, cultural y económica de la profesión. La sociedad obliga día a día a formar diseñadores integrales conscientes de la realidad. Sin embargo, como lo resalta Raúl Belluccia (2007), se debe recordar que "el diseño es una práctica profesional, un hacer y no una teoría. Pues de forma extraña y paradójica - hoy - hay escuelas de diseño en donde se diseña muy poco o lo que es peor, hay cada vez más escuelas de diseño que enseñan menos diseño" (p. 33). Por ello es preocupante y ocupante la práctica responsable de su enseñanza, recuperando el sentido de compromiso y la responsabilidad en la construcción personal y profesional de los futuros diseñadores.

El diseñador Roberto Osses (2014), propone cuatro ejes fundamentales que deben constituir a todo diseñador: sociedad, cultura, identidad y patrimonio. Asimismo resalta la importancia de trabajar en su desarrollo integral pues será imposible que puedan desenvolverse en el mercado, sino conocen ni comprenden profundamente a la sociedad. El diseñador gráfico no es solo un reproductor de ideas que se plasman en una imagen, es un profesional que requiere muchas habilidades cognitivas que le permitan estar en constante aprendizaje y adaptarse a los constantes cambios de la sociedad y sus necesidades. Para Mafla (2011), el perfil del diseñador va más allá, ya que no solo construye conceptos, sino que transforma a la sociedad y la manera en la que las personas se ven a sí mismas; el mismo investigador menciona que: "la responsabilidad del diseñador con la humanidad es amplia, no solo crea objetos, tecnologías, experiencias, sino ambientes que afectan la globalidad de nuestros sentidos" (párr. 1).

Entonces nuevamente en el plano de la formación y la función de las escuelas, se advierten fallas en la concepción de la profesión al interior de muchos de los programas de estudio. 
Se está haciendo una costumbre que el alumno "diseñe" o, más bien ejecute por el simple hecho de hacer y que se despegue del pensar de la mano de la ejecución. Adicionalmente se los está sobrecargando de habilidades, adelantadas a su momento o a su madurez creativa. Con esto se prioriza la tecnología sin considerar si los estudiantes han alcanzado la suficiente madurez y conciencia, y se están mecanizando como operarios y no como profesionales.

Paradójicamente en las escuelas hay una mayor preocupación por la tecnología, más que por el propio diseño. En general, el interés se centra en la "forma" o resultado final de los objetos o productos gráficos solicitados a demanda de un programa y no como respuesta a un proceso formativo integral. Se ha olvidado que la clave para que el diseñador sea verdaderamente un profesional consiste en entender que su papel es producir significados, entendiendo éstos como fenómenos culturales. Cada día cuesta más asimilar que como profesionales, sólo inmersos en la realidad cultural se puede interpretarla y representarla. No es posible crear sin conocer, profundizar y convivir. Considerando lo anterior, es Joan Costa quien al referirse a los nuevos paradigmas en el ámbito del diseño y la comunicación visual afirma categóricamente:

Debemos centrarnos en el hecho de que el diseño establece un sistema de relaciones constantes entre nosotros y los ambientes que ocupamos, los objetos que utilizamos y los mensajes que integramos. Y así, los productos del diseño forman parte de nuestra cultura. (2014, p.89)

En este orden de ideas, el diseño se constituye como una actividad mediadora que permite configurar el entorno artificial, asimilándose como un elemento significativo que regula las interacciones de los indivuduos en la sociedad y con la sociedad. Y en la medida que su fin último sea mejorar el hábitat, resolver problemas y en general, mejorar la calidad de vida, la esencia del diseño cumple una función informativa y comunicativa, pero sobre todo una función social. De esta manera se constatan elementos distinguibles y evidentes, como la intencionalidad y la significación. Lo que define a los mensajes elaborados a partir del diseño es asumirlos como "constructos humanos y productos culturales".

En estos tiempos hipermodernos, Lipovetsky (2006) precisa que:

Hemos pasado, para bien y para mal, a la era "hipermoderna" que deja atrás todo "pos" y todo "ismo". Ahora todo es "hiper" y paradójico. Los individuos hipermodernos están más conscientes de la necesidad de comportamientos responsables, pero en sus primeros pasos son muy irresponsables, están mejor informados, y más desestructurados, son adultos inestables, más abiertos, pero más influenciables, más críticos, pero a la vez más superficiales, más escépticos y por supuesto, menos profundos. (p.59)

Tal y como se afirmaba al inicio del texto, cada día llegan a las aulas jóvenes aparentemente mejor informados y con una habilitación tecnológica importante, pero que carecen de estructuras fundamentales de pensamiento, decisión y acción. Derivado de esta postura, Sandra Navarrete (2014), afirma que: 
Actualmente se observan con claridad dos actitudes muy definidas frente al modo de diseñar: por un lado están los diseñadores que utilizan su formación teórico-crítica como fuente de inspiración, y por otro lado están los que creen que la genialidad es algo innato, consideran que el conocimiento entorpece el proceso creativo, buscan en diferentes estímulos, de origen misterioso, la fuente de las ideas geniales. (p. 25)

Se reduce la intervención y carácter del diseño a su capacidad para elaborar soluciones sin sentido, más cercanas a la moda y la inmediatez y alejas del trasfondo real que debe asumir el carácter profesional de la disciplina.

En aras de hipermodernizar y asociar al diseñador con el clisé que marca la tendencia del siglo XXI, en los salones de clase se pierde el rumbo de la brújula. En primer lugar, porque se olvida la responsabilidad que se tiene en la formación de los diseñadores. La memoria falla, desde el enfoque acerca de qué se está diseñando, pero también de cómo se está diseñando, cómo se gestionan estos diseños, qué conciencia se tiene acerca de los intereses de para quién se diseña, y si existe un compromiso responsable con los efectos, sociales, económicos, políticos, culturales y ambientales que generan estos diseños, desde la perspectiva de su producción, su uso y desuso.

En torno a estas afirmaciones, Bill Buxton (en Cano, R., 2018) refiere que:

Simplemente no es aceptable adoptar la actitud de que solo hacemos las herramientas, no siendo además responsables de cómo se usan. La realidad es que el diseño puede tener un gran impacto en el recorrido de ciertos caminos. Aquellos de nosotros que diseñamos cosas debemos hacer los mejores esfuerzos para asegurarnos de que esos caminos se ajusten a nuestra brújula ética. Por supuesto, eso implica que debemos ser conscientes de cuáles son nuestros valores. (Cano, 2018, s/n)

Por ello se ha insistito a lo largo de este documento no sólo en las responsabilidades individuales de cada estudiante, también en las de las escuelas en la formación del profesional del diseño y las implicaciones de su hacer. En definir de forma acertada procesos, estrategias y modelos que se ocupen aún más de transmitir no solo conocimientos y habilidades, sino actitudes y valores propios de esta profesión y principalmente buscando blindar las filas, para despegarla de estereotipos y clisés muy lejanos de lo que es el Diseño, pues como Papanek (1971) afirmaba:

La responsabilidad del diseñador (...) su buen juicio social y moral tiene que entrar en juego mucho antes de que empiece a diseñar, porque tiene que juzgar, apriorísticamente, además si los productos que se le pide que diseñe o rediseñe merecen su atención o no. En otras palabras, si su diseño estará a favor o en contra del bien social (p.57). 
Aunado a todo esto, nuevamente de forma paradójica y citando a Norberto Cháves (2013), "el pragmatismo acrítico sigue empobreciendo la conciencia social y cultural de los profesionales. Lo que plantea un eje neurálgico, que apunta hacia la formación de los profesionales del diseño" (p. 31). Es imposible crear, diseñar o formular una propuesta congruente con el conjunto de problemas de comunicación que la sociedad plantea, si el profesional del Diseño sigue pensando como ente aislado; la única manera es entender que forma parte de un contexto, que comparte conocimientos y aptitudes; que la forma como siente y percibe el mundo ha sido asimilada poco a poco del contexto cultural que es el de la sociedad a la cual pertenece y que su trabajo tiene repercusiones en ella.

El papel de las escuelas y universidades es medular. No solo en la transmisión de los distintos saberes, incluidos aquellos que trastocan la formación integral de la persona, también en la concepción social de perfil profesional y en la gestión del concepto de cada profesión, como concepto generalizado y como autoconcepto, relacionado con la concienca individual.

De esta manera y para la obtención de resultados óptimos y más aproximados a un diseño con responsabilidad social, habría que enfocarse en los procesos de aprendizaje de los futuros profesionales del diseño en las universidades, pasando por saberes y criterios de la misma universidad y, posteriormente, por aquellos que se vierten en los programas de estudio de las distintas licenciaturas en diseño. La escuela es partícipe de las realidades sociales y debe buscar, abordar y atender las problemáticas de un país y, a partir de ello, situar sus compromisos sobre la formación profesional, en vías de una transformación del contexto social. Parsons (citado en Dingwall, 2004) dice que:

las profesiones no son sólo las portadoras del conocimiento científico, también son guardianes seculares de lo sagrado, el sacerdocio del mundo moderno; pero un sacerdocio que reconoce lo incognoscible de las cosas, que se enfrenta a las incertidumbres y reconoce la apertura del mundo cambiante (p. 10).

Hoy, como lo plantean Reyes y Pedroza, los diseñadores en el mundo de las profesiones deben permacer en constante cambio, continuar reinventándose siempre. El diseñador "debe deconstruirse, construirse y reconstruirse al mismo ritmo que lo hace la ciencia, la tecnología y el desarrollo humano.” (Reyes y Pedroza, 2015, p.16).

Como individuo creativo, responsable y comprometido, debe proponer siempre nuevos modos de ser, hacer, y también de pensar, nunca mecanizarse o permancer estático. "Como sujeto, formado profesionalmente, deber proyectar su propio background, y en esa propuesta se proyectará a sí mismo. Sus intereses, sus capacidades creativas, pero también su propia personalidad. Como ser humano, propulsor de la cultura debe ser capaz de crear realidades y experiencias que conlleven a una cultura divergente, novedosa, en renovación constante". (Reyes y Pedroza, 2015, p.16).

En esta hipermodernidad soportada por tres componentes esenciales: el mercado, la eficiencia técnica y el individuo, resulta fundamental, replantear la ética de la responsabilidad profesional. El momento actual se distingue por la acelerada transformación en todos los campos del conocimiento y del quehacer humano. También se distingue por la desinformación, una paradoja más de la sociedad de la información, o por la sobrecarga de ella, 
que termina sin hacerle sentido al estudiante o profesional, que carente de procesos de pensamiento más estructurados, responsables y conscientes, recurre a mecanismos poco éticos en su práctica diaria.

Se acostumbra asumir acciones, ideas y formas que no responden a determinados patrones culturales considerados propios, sino a culturas diferentes, como dotados de falta de autenticidad de las soluciones. El principal cuestionamiento residiría no tanto a su origen externo, sino al hecho de ser repetidas sin reflexión, ni crítica. Se está fomentando una cultura de la imitación que como afirma Villoro: “... no es inauténtica por dejarse influir por elementos externos a la 'realidad', sino por aceptarlos sin ponerlos en cuestión integrándolos a nuestros deseos y necesidades reales" (1965, p. 161). Y desafortunadamente, no todos los diseñadores tienen claro qué valores y demandas éticas conllevan tales planteamientos a la hora de diseñar.

Por todo lo anterior, se puede entender el diseño con responsabilidad social como las respuestas creativas, coherentes y pertinentes que genera el diseñador con las comunidades a las cuales dirige sus esfuerzos, y que tiene como objetivo lograr transformaciones que contribuyan en la construcción de una sociedad más justa y equitativa.

El diseño, como proceso clave de la comunicación, necesita de comportamientos éticos para ser útil a la sociedad. Los diseñadores reivindican el valor de la profesión, no sólo en el terreno de las empresas, en el ámbito económico o como actividad inserta en los procesos productivos, sino desde un ámbito cultural. En este tenor, vale la pena recuperar el acento que pone Luz del Carmen Vilchis (2015) en ello, cuando afirma que:

Una de las tareas de los diseñadores en la cultura es la construcción y permanencia de lo humano y de los valores que le dan sentido a la vida a partir de la comunicación. Des-construir significa des-culturizar; construir, por el contrario, implica ensalzar, acentuar y multiplicar la cultura; la vida de un diseñador gráfico es una responsabilidad ante el vacío y la esterilidad del conocimiento y de la masividad de la comunicación. (párr. 41)

El diseño está destinado a la sociedad y por tanto es capaz de generar y transmitir valores culturales y éticos. Trabajar o no bajo estos principios es una decisión que se toma y asume todos los días, que influye en las personas y que suma o resta en la cuenta de resultados, individuales de cada profesional y colectivos de cada profesión.

El diseño dejó de ser un oficio para convertirse en una profesión, con grandes alcances en este siglo, ampliando su perfil profesional y laboral justamente por la funcionalidad que es inherente a la disciplina, y la tecnología que es parte de la cotidianeidad y sus procesos de diseño y proyección. 


\section{Conclusiones}

Los cambios vertiginosos en materia tecnológica demandan de los diseñadores una gran capacidad de adaptabilidad, y un estado constante de aprendizaje. El trabajo interdisciplinario ha sido un factor determinante para la evolución y desarrollo del diseño. Se viven días en donde el acceso a una gran cantidad de información, la transmisión y discusión de la misma, han puesto en jaque la estructura de la profesión y de muchas otras más.

Por ello, se necesitan reconfigurar las discusiones éticas, que históricamente son parte del diseño. En este transfondo debe pujarse por una nueva ética del diseño. La visión del futuro considera e involucra a diseñadores que trabajen en muchos campos profesionales, como agentes de cambio, que sean consientes y responsables de los efectos de su quehacer, en aras del bienestar humano. Profesionales responsables con una dirección clara, "diseñadores ciudadanos" como los define Margolin, cuyo rol social alcance dimensiones incluso políticas. Se trata en definitiva de descontextualizar al diseño como una práctica productiva profesional aislada y solo concebible de la mano de las tecnologías y recontextualizarla en sus dimensiones social y cultural.

Los problemas específicos de estos tiempos se deben, no al progreso tecnológico en sí mismo, sino a la ausencia de un progreso simultáneo en la dimensión filosófica y humana de los que habitan el mundo. Las verdaderas soluciones para este momento histórico no son resolver los problemas ético-sociales a posteriori, cuando ya se ha producido el daño sino, en la medida de lo posible, hacerlo a priori, recuperando la capacidad de prever las consecuencias a largo plazo.

Como se puede leer en una reflexión interesante en la revista Paredro (2013): "la solidez de la profesión del diseño se la debemos al esfuerzo de muchos profesionales que han transmitido su saber en el campo de la creatividad e innovación", pero también no se debe ignorar el momento que se está viviendo y todos los problemas a los que actualmente se enfrenta esta profesión, que puja entre el reconocimiento o el total desconocimiento de los que realmente se forman como diseñadores y todos aquellos que adquieren tan solo destreza técnica o simplemente, la habilidad tecnológica y que invaden los espacios y puestos que un diseñador debiera ocupar.

En las escuelas y universidades, deben reforzarse aspectos relativos a la cultura, la identidad y los valores éticos. La responsabilidad profesional es el deber ser, que está en la persona, pero que se perfecciona y se fortalece cada día en el ejercicio de la misma y es una decisión, que va más allá de códigos, normas o leyes aprendidas de memoria. La formación integral del diseñador le permitirá ejercer un papel más estratégico y fortalecer su toma de decisiones garantizando con ello resultados efectivos al generar sus propuestas; a la par, tendrá la posibilidad de reflexionar en torno al propio proceso de diseño, los valores que su trabajo refleja, así como el impacto tanto social, como económico, ambiental y cultural de los proyectos que entrega a la sociedad.

Ser diseñador implica un compromiso con una serie de valores, que empiezan por uno mismo, que se adquieren junto con los saberes y conocimientos propios de la profesión, pero que transcienden más allá de la persona. Acordar estas normas y responsabilidades desde las escuelas, no en lo individual, sino en lo colectivo, reforzará las relaciones con 
clientes y usuarios y de esta manera los diseñadores en ejercicio, los docentes y aquellos futuros diseñadores que se encuentran en formación, contribuirán a construir un tejido colectivo que ayudará a difundir de manera correcta la cultura del diseño, sus alcances y dimensiones y el valor de la profesión en la sociedad.

En síntesis, la profesión del diseño lleva entremezcladas dos cuestiones, por un lado su inminente redefinición constante y por el otro, sólo puede asumirse como diseñador no el que tiene el oficio, sino la vocación.

\section{Referencias}

Actas de Diseño (2006). Año I, No. I. Universidad de Palermo.

Belluccia, R. (2007). El Diseño Gráfico y su enseñanza. Paidós.

Cano, R. (2018). Importancia de la Ética del Diseño, Consultado el 13 de enero, https://www. researchgate.net/publication/329423758_IMPORTANCIA_DE_LA_ETICA_EN_EL_DISENO/citation/download.

Catálogodiseño (1 de septiembre de 2014). Entrevista a Roberto Osses. Consultado el 5 de noviembre de 2014, de: http://www.catalogodiseno.com/2014/09/01/roberto-ossestipografo-chileno/

Chaves, N. (2006). Intentando dispersar la bruma. Actas de Diseño ํo1, Agosto 2006, Universidad de Palermo.

Chaves, N. (2013). El Oficio de Diseñar. Gustavo Gili.

Costa, J. (2014). Diseño de Comunicación Visual: el nuevo paradigma, Revista Grafica, Vol. 2, Núm. 4, Consultado el 11 de febrero de 2020, https://revistes.uab.cat/grafica/article/ view/v2-n4-costa

Cortina, A. (2000). “Presentación”, en Adela Cortina y Jesús Conill (coords.), 10 palabras clave en ética de las profesiones. Verbo Divino.

Cortina, A. (2009). Cuadernos de la cátedra de ética. U. Iberoamericana-León.

Cortina, A. (2001). Ética mínima. Introducción a la filosofía práctica. Tecnos

Dingwall, R. (2004). Las profesiones y el orden social en una sociedad global. Revista Electrónica de Investigación Educativa, Vol. 6, No.1. Universidad Autónoma de Baja California, Consultado el 7 de noviembre de 2019. https://redie.uabc.mx/redie/article/view/95/165

Gutiérrez, M. (2013). Mucha Tecnología y poco diseño, Foro Alfa. https://foroalfa.org/ articulos/mucha-tecnologia-y-poco-diseno.

Hortal, A. (2002). Ética general de las profesiones. Desclée de Brouwer.

Lévy, P. (2004). Inteligencia Colectiva, por una antropología del ciberespacio. Biblioteca Virtual Em Saudé; BIREME-OPS-OMS.

Lipovetsky, G. (2006). Los tiempos hipermodernos. Colección Argumentos Anagrama.

Mafla, J. (28 de septiembre de 2011). El diseñador como modelador cultural, Foro Alfa. Consultado el 20 de enero de 2020. http://foroalfa.org/articulos/el-disenador-comomodelador-cultural. 
Manual de Buenas Prácticas del Diseño 3(2009). La profesión del diseño. Surgenia, Centro Tecnológico Andaluz de Diseño, Córdoba, España.

Margolin, V. (1991). Los estudios de diseño y la educación de los diseñadores, Pedagogía del diseño. Gustavo Gili.

Margolin, V. (2011). Diseño y el Riesgo del Cambio, Revista KEPES Año 8 No. 7 enerodiciembre 2011. http://vip.ucaldas.edu.co/kepes/downloads/Revista7_7.pdf

Margolin, V. (2017). Construir un mundo mejor. Diseño y reponsabilidad social. Designio.

Miranda, S. (2009) "Prólogo" Manual de Buenas Prácticas del Diseño 3. La profesión del diseño. Centro Tecnológico Andaluz de Diseño.

Navarrete, S. (2014). Abstracción y expresión. Una reflexión de base filosófica sobre los procesos de diseño, Cuadernos de Diseño No. 49. Universidad de Palermo.

Osses, R. (2014) Catálogodiseño del 1 de septiembre de 2014.

Papanek, V. (1971). Diseñar para el mundo real, ecología humana y cambio social. Blume Ediciones.

Reyes, A. y Pedroza, R. (2015). Profesión y profesionalismo en el Diseño Industrial. M.A. Porrúa.

Reyes, A. y Pedroza, R. (2017). Retos de la Formación profesional del diseñador industrial en la Cuarta Revolución Industrial, (4RI). Prensa.

Soto M. y Meléndez M. (2008). El diseño: Del oficio a la profesión. Actas de Diseño $N^{\circ} 4$ Año II, Vol. 4. Universidad de Palermo.

Vilchis, L. del C.(2015). Responsabilidad social del diseño, Inventio, Vol. 11, No. 25. Universidad Autónoma del Estado de Morelos, Consultado el 10 de enero de 2020, http:// inventio.uaem.mx/index.php/inventio/article/view/109/189

Villoro, L. ( 1965-1966), "Historia de las ideas", Historia Mexicana, vol. XV, núms. 58-59, México: El Colegio de México, Consultado el 11 de enero de 2020, https://www.jstor. org/stable/25134577?read-now=1\&seq=1\#page_scan_tab_contents

Abstract: In a context of professional practices in mutation, there is no profession that remains static to technological change. In the case of Design, its conception, foundation and action have been disrupted. This process and the need to transform the values of designers, also require to modify the way of assuming the professionalization of the discipline and to pay attention to the epistemological problem that raises the issue of professional identity, as representation and self-concept.

In a world where everything is "hyper", as Lipovetsky says (2006), hypermodern individuals, aspiring designers, may be better informed and have access to knowledge immediately, are predisposed to technology and "preloaded" with a large number of skills, although in reality they have great deficiencies. Paradoxically, the social and cultural conscience of the profession has been impoverished, to give way to a pragmatism dissociated from responsibility and associated with novelty or fashion. 
This reflection seeks to investigate the values, identity and professional ethics of Design. It analyses the orientation or reorientation of the training processes and the importance of ethics, to return the designer as a subject belonging to the social system that, by intervening in it professionally, modifies or renews it.

Keywords: design - ethics - identity - professional - values

Resumo: Num contexto de práticas profissionais em mutação, não há profissão que permaneça estática à mudança tecnológica. No caso do Design, a sua conceção, fundação e ação foram interrompidas. Este processo e a necessidade de transformar os valores dos designers, exigem também modificar a forma de assumir a profissionalização da disciplina e prestar atenção ao problema epistemológico que levanta a questão da identidade profissional, como representação e auto-conceito.

Num mundo onde tudo é "hiper", como diz Lipovetsky (2006), indivíduos hipermodernos, aspirantes a designers, podem estar mais bem informados e ter acesso ao conhecimento imediatamente, estão predispostos à tecnologia e "pré-carregados" com um grande número de competências, embora na realidade tenham grandes deficiências. Paradoxalmente, a consciência social e cultural da profissão tem sido empobrecida, para dar lugar a um pragmatismo dissociado da responsabilidade e associado à novidade ou à moda.

Esta reflexão procura investigar os valores, identidade e ética profissional do Design. Analisa a orientação ou reorientação dos processos de formação e a importância da ética, para devolver o designer como um assunto pertencente ao sistema social que, ao intervir nele profissionalmente, o modifica ou renova.

Palavras chave: design - ética - identidade - profissional - valores

[Las traducciones de los abstracts fueron supervisadas por el autor de cada artículo] 